\title{
\&IIIBRAGEC

\section{PLATAFORMA DE GESTÃO DE RESÍDUOS PARA O FOMENTO DA ECONOMIA CIRCULAR NA CONSTRUÇÃO CIVIL ${ }^{1}$}

\section{BARRETO NETO, João Félix (1); COSTA, Milena Mota (2); ALBERTE, Elaine Pinto Varela (3); CARNEIRO, Alex Pires (4)}

(1) Universidade Federal da Bahia, joao.felix@ufba.br (2) Universidade Federal da Bahia, milenamc@ufba.br, (3) Universidade Federal da Bahia, elaine.varela@ufba.br (4) Universidade Federal da Bahia, alexpires@ufba.br

\begin{abstract}
RESUMO
Existe uma carência atual por ferramentas de gestão que garantam ao gerador de resíduos de construção e demolição (RCD) o controle e rastreabilidade das ações tomadas, segundo a Lei $n^{\circ}$ 12.305:2010. Por outro lado, a reinserção de resíduos ao ciclo produtivo da construção é necessária para o fomento da economia circular no setor. Este artigo apresenta uma proposta de plataforma on line que promove e controla a gestão, compra e venda de RCD entre os agentes pertencentes à cadeia produtiva do setor, a partir do uso da tecnologia de registro blockchain. Inicialmente, realizou-se uma revisão da literatura para identificação do panorama atual do processo e das tecnologias aplicáveis; e o levantamento de dados em uma construtora para identificação dos processos, fluxos de informação, ferramentas, dificuldades e boas práticas. O planejamento da estrutura e formato da plataforma foram definidos a partir das informações levantadas. Através de um conceito SaaS (Software as a Service), a plataforma desenvolvida permite fortalecer a conexão entre stakeholders para a busca de soluções sustentáveis para o gerenciamento de RCD. Estas soluções independem do tamanho ou segmento da empresa, e permite a criação de oportunidades de negócios dentro da própria indústria que proporcionem práticas de fomento à economia circular como logística reversa, servitização e simbiose industrial.
\end{abstract}

Palavras-chave: Blockchain, Economia circular, Gestão de resíduos, Construção civil.

\begin{abstract}
There is a current lack of management tools that guarantee the construction and demolition waste (CDW) generator the control and traceability of the actions taken, according to Law No. 12,305:2010. On the other hand, the reinsertion of waste to the construction productive cycle is necessary to foster the circular economy in the sector. This article presents a proposal for an online platform that promotes and controls the management, purchase and sale of CDW among agents belonging to the sector's production chain, using blockchain registration technology. Initially, a literature review was carried out to identify the current panorama of the process and the applicable technologies; and data collection in a construction company to identify processes, information flows, tools, difficulties and good practices. The planning of the structure and format of the platform were defined based on the information collected. Through a SaaS (Software as a Service) concept, the developed platform allows to strengthen the connection between stakeholders in the search for sustainable solutions for the management of CDW. These solutions are independent of the size or segment of the company, and allow the creation of business opportunities within the industry itself that provide practices to foster the circular economy such as reverse logistics, servitization and industrial symbiosis.
\end{abstract}

Keywords: Blockchain, Circular Economy, Waste Management, Construction.

\footnotetext{
1 BARRETO NeTO, J. F.; COSTA, M. M.; ALBERTE, E. P. V.; CARNEIRO, A. P. Proposta de uso da tecnologia de registro Blockchain na gestão de suprimentos da Construção Civil. In: SIMPÓSIO BRASILEIRO DE GESTÃO E ECONOMIA DA CONSTRUÇÃO, 12., 2021, Maceió. Anais[...] Porto Alegre: ANTAC, 2021. p.1-8. Disponível em:

https://eventos.antac.org.br/index.php/sibragec/article/view/450. Acesso em: 2 out. 2021.
} 


\section{INTRODUÇÃO}

O crescimento econômico associado a uma população global em constante ascensão tendem a acelerar o uso de recursos e produzir um desequilíbrio ambiental no mundo. No âmbito da construção civil, este cenário é destacado visto a grande quantidade de resíduos produzidos. ABRELPE (2019) indica que a geração deste resíduo aumentou 35\% nos últimos 10 anos, atingindo 44,5 milhões de toneladas em 2019, cerca de 56\% dos resíduos gerados no país no referido ano.

Neste contexto, a economia circular (EC) possui alto potencial para minimizar as questões ambientais do setor da construção. Suas premissas buscam otimizar recursos e reduzir a geração de resíduos através de oportunidades de mercado e crescimento econômico, com a implementação de novas tecnologias, processos, serviços e modelos de negócios (KORHONEN et al., 2018).

Entende-se aqui EC como um modelo econômico que busca permitir a manutenção dos materiais no ciclo produtivo através da sua reutilização, recuperação, reparação e reciclagem, propiciando maior eficiência na gestão de recursos, sustentabilidade e bem estar da população (RIBEIRO e KRUGLIANSKAS, 2014; GHISELLINI et al, 2015). E para tal, deve-se considerar uma abordagem completa do ciclo de vida da edificação, desde a extração da matéria prima até a destinação dos resíduos, passando pelas etapas de manufatura, construção, manutenção e revitalização do ambiente construído (CONAMA, 2018).

Diversas práticas podem ser adotadas para implementação de principios da EC na construção ao longo do ciclo de vida da edificação: ecodesing - metodologia para desenvolvimento de produtos sustentáveis; avaliação do ciclo de vida (ACV) - ferramenta que avalia os impactos ambientais de um produto ou serviço em todas as suas etapas (extração, produção, distribuição, uso e fim da vida); servitização -=- estratégia de incorporação do modelo de uso ou serviço em detrimento do modelo de propriedade, possibilitando um uso mais inteligente dos recursos; reutilização no local - solução para minimizar o consumo de matérias primas e reduzir as distâncias de transportes de materiais; manutenção - trabalhos realizadas durante a etapa de uso do produto, com o objetivo de garantir os níveis mínimos de qualidade; reabilitação parcial - intervenções em elementos do sistema em função do estado de deterioção, mantendo a identidade e contexto; reabilitação total - reestruturação completa do sistema em função do estado de deterioção, mantendo a identidade e o contexto; desconstrução - desmantelamento cuidadoso, visando recuperar ao máximo materiais e componentes do sistema; valorização energetica - utilização do poder calorífico dos resíduos; entre outros (CONAMA, 2018).

Por outra parte, a baixa e lenta adesão às novas tecnologias agrava o processo de gestão de $\mathrm{RCD}$, visto que a indústria da construção é considerada uma das mais artesanais no panorama mundial (KOUHIZADEH, 2020). E segundo Kouhizadeh (2020), Wang (2016), Conforto (2019) e Roque (219), parte dos dilemas relacionados ao fomento da EC podem ser solucionados através de tecnologias da informação e comunicação (TICs). Assim, estudos que visem o desenvolvimento de modelos de gestão mais sustentáveis e com uso mais intensivo de TICs devem ser fomentados.

Nessa pespectiva, a tecnologia de registro Blockchain (ou sua sigla em inglês BT, Blockchain tecnology), se destaca por se tratar de uma inovação disruptiva que permite o compartilhamento e rastreamento de informações em redes de fornecimento (Kouhizadeh, 2020). BT pode ser definido como um banco capaz de replicar, compartilhar e sincronizar dados espalhados por diferentes locais através de uma rede distribuida. Possui forte potencial para impulsionar iniciativas econômicas circulares, visto que a mesma age como uma facilitadora que pode moderar as inter-relações trazendo mais transparência para os processos que envolvem a gestão de resíduos e minimizando a necessidade de desintermediação nas transações (KOUHIZADEH, 2019). BT proporciona 
decentralização, segurança, imutabilidade, auditabilidade, confiabilidade, transparência, desintermediação e veracidade no processamento de dados (CONFORTO, 2019), sendo composta por: livro razão - um livro no qual são realizados registos; rede de pares transmite mensagens ordenadas, de modo a armazenar, atualizar e manter o livro-razão; serviço de associação - ordena, organiza e transmite as transações recebidas, garantindo o controle de acesso à blockchain; contrato inteligente - coleção de códigos e datas implantado para assinar transações na rede blockchain; evento - confirmação de um novo bloco na rede (DIAS, 2019); gerenciamento de sistemas - cria, modifica e monitora o sistema; e sistema integrado - integrar a blockchain com sistemas externos visando reconhecer (assumir) outras capacidades; gerenciamento de sistemas - monitora o estado do livro razão distribuído entre os nós através da comunicação da rede de pares (NGUYEN et al. 2018); e sistema integrado - integração direta a blockchain com sistemas externos de forma eficiente visando desintermediar processos (XU et al 2016).

Esse estudo tem como objetivo propor uma plataforma de gestão de resíduos para fomento da EC no setor da construção civil através do uso de BT. Espera-se que o sistema proposto impusione novos modelos de negócio capazes de conduzir os metodos de produção a um novo fluxo de resíduos, alinhado com os princípios da EC, visando contribuir para a promoção de atividades economicamente circulares na construção.

\section{METODOLOGIA}

Este estudo, de caráter exploratório, propõe uma estrutura de plataforma on line para controlar a gestão, compra e venda de RCD entre os agentes pertencentes à cadeia produtiva do setor, a partir do uso da tecnologia de registro blockchain. A proposta desenvolve-se a partir de revisão sistemática da literatura (RSL) sobre o tema e da analise sobre os processos e práticas típicos de uma construtora. Considera-se aqui RSL como um método para identificar estudos que analisam determinada questão de pesquisa, avaliando os resultados de modo a identificar conclusões sobre um corpo de conhecimento (HULLEY et al., 2015). O estudo divide-se em três macro etapas (Figura 1).

Figura 1 - Estrutura metodológica adotada
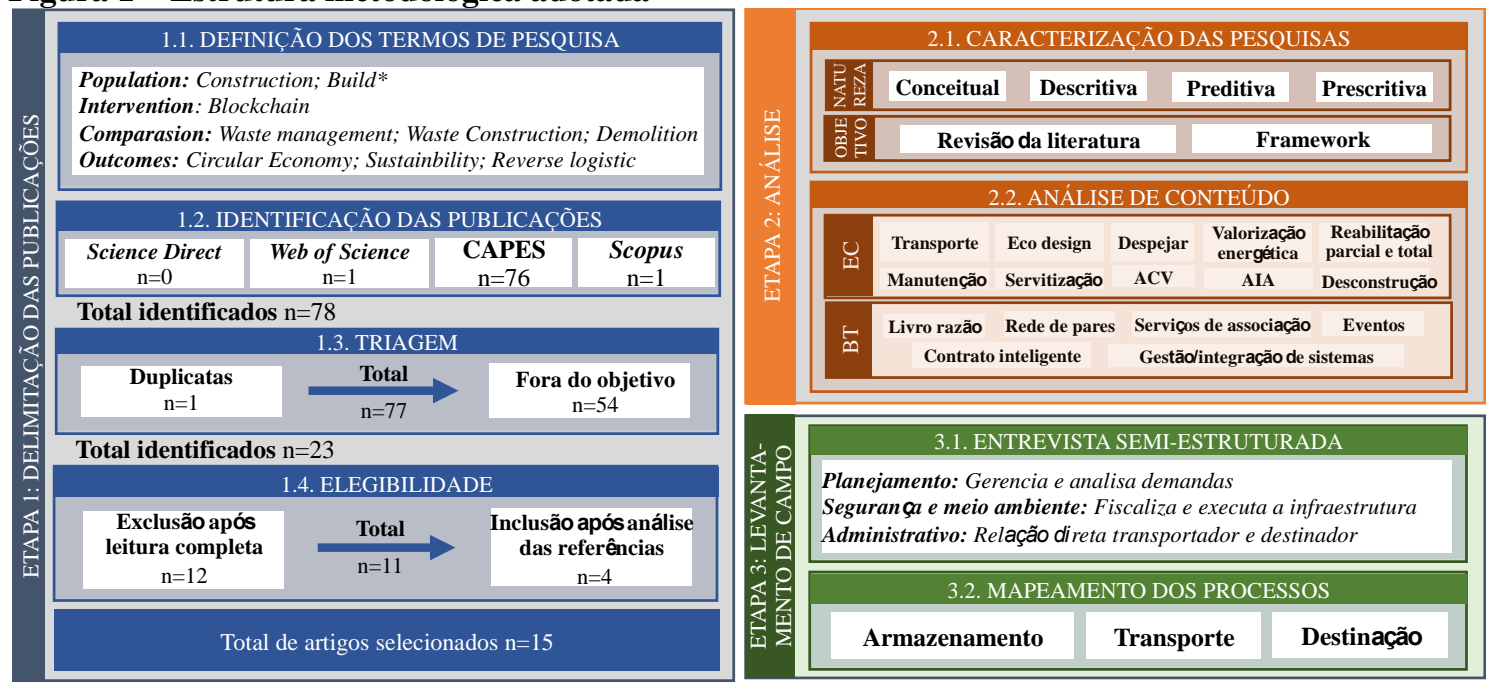

Fonte: autores

Na Etapa 1 (Delimitação das publicações) definiu-se termos (strings) de pesquisa, com base na adaptação feita por Agostinho e Granja (2016) do método PICO (Population, Intervention, Comparasion e Outcomes). Os termos de pesquisa foram definidos segundo assunto (population), intervenção (intervention), comparações (comparasion) e efeitos (outcomes) (PAI et al., 2004). Na busca das publicações, foram utilizados os seguintes bancos de dados: Science Direct, Web of Science, Periódicos CAPES e Scopus. Para seleção das publicações, identificou-se os artigos de maior relevância para o tema. 
Realizaram-se dois processos de seleção. O primeiro filtro (triagem) foi realizado a partir da leitura do título e do resumo de cada publicação, buscando-se confirmar a relação do tema tratado no artigo com a questão da pesquisa. O segundo filtro (elegibilidade) analisa através de leitura completa se efetivamente o artigo tem relação direta com a questão da pesquisa.

Na Etapa 2 (Análise), realizou-se análise estruturada do conteúdo dos artigos, a partir da sua leitura completa e detalhada. Além da caracterização das pesquisas por natureza e objetivo, buscou-se identificar evidências sobre a aplicação da BT para apoiar processos da economia circular. Essas evidências foram destacadas e categorizadas em função seu objetivo e forma de fomento à EC. Tais evidências (codificadas neste documento como $\left.E_{n}\right)$ também foram analisadas a partir de matriz de análise de conteúdo, quando foi possível destacar a conexão / relação existente em cada evidência entre os componentes da BT (segundo Dias, 2019) com as funções / processos da economia circular aos quais a evidência está vinculada (segundo CONAMA, 2018). Os fatores analisados nesta etapa são apresentados na figura 1 .

Na Etapa 3 (Levantamento de campo) foi identificada uma empresa atuante no segmento de construção com 20 anos de antiguidade, grande porte, e experiência na construção de obras comerciais; coorporativas; e de infraestrutura. A empresa se destaca por forte atuação em obras públicas e por possuir certificações ISO 9001e PBQP-H (Programa Brasileiro da Qualidade e Produtividade do Habitat). Além disso, possui setor de sustentabilidade estruturado. O levantamento dos dados foi realizado por observações de campo e entrevistas semi estruturadas com a equipe técnica da empresa, de modo a se realizar o mapeamento dos processos de gestão de resíduos; identificar os fluxos de informação relacionados; e as boas práticas e dificuldades existentes.

Com base na análise conjunta das informações obtidas nas etapas 2 e 3 , foi desenvolvida uma proposta de plataforma com formato e estrutura que atendessem as demandas do setor, considerasse as potencialidades da tecnologia objeto de estudo, e as boas práticas identificadas na literatura.

\section{ANÁLISE DOS RESULTADOS}

\subsection{Análise de conteúdo}

A Figura 2 apresenta as principais características da bibliografia selecionada.

Figura 2 - Bibliografia selecionada

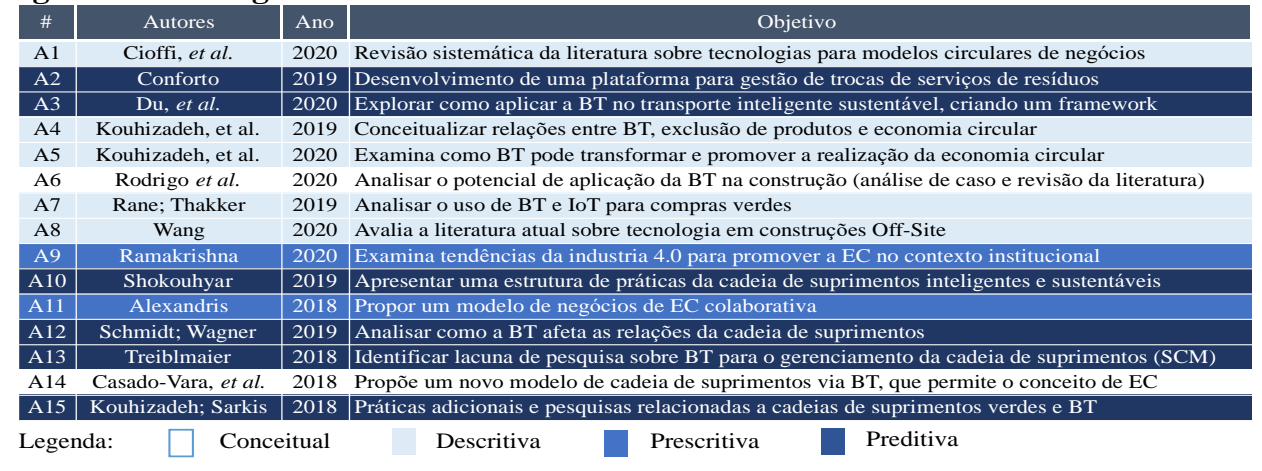

Fonte: autores

A partir dela, nota-se que boa parte da bibliografia analisada (40\%) possui carácter preditivo (objetivam apresentar e/ou explorar o uso da BT, prevendo resultados e tendências futuras). Os de natureza descritiva (33\%), por sua vez, se propõem a analisar de forma analítica o uso da BT e o panorama atual. Finalmente, os artigos conceituais buscam analisar o potencial de uso da BT e propor novos modelos de aplicação através da observação e análise das informações existentes, enquanto os prescritivos visam 
examinar as tendências e propor modelos de negócios, por meio de analises das consequências futuras de ações tomadas.

Trinta e duas evidências sobre a aplicação de BT para fomento da EC foram identificadas nos artigos selecionados. Tais evidências foram codificadas (En), e categorizadas por artigo e objetivo (Figura 3). Boa parte das evidências (16) estão relacionadas ao fomento da EC por melhor gestão das informações (GI). Nesse âmbito, contudo, o uso da BT pode ser aplicado para distintos e variados fins: ACV, gestão da construção como banco de materiais, gestão de valor dos materiais, gestão de estoque, e desempenho do stakeholder, dentre outros. Os objetivos vinculados a transporte e rastreamento ( $\mathrm{T}$ e R), por outro lado, destacam-se com 17 evidências, ao total. Nestes casos, evidencia-se o uso da BT para monitoramento dos fluxos de processos / produtos, de um modo geral, indicando que o forte potencial da tecnologia para tal fim.

Figura 3 - Evidências identificadas por código, artigo e objetivo

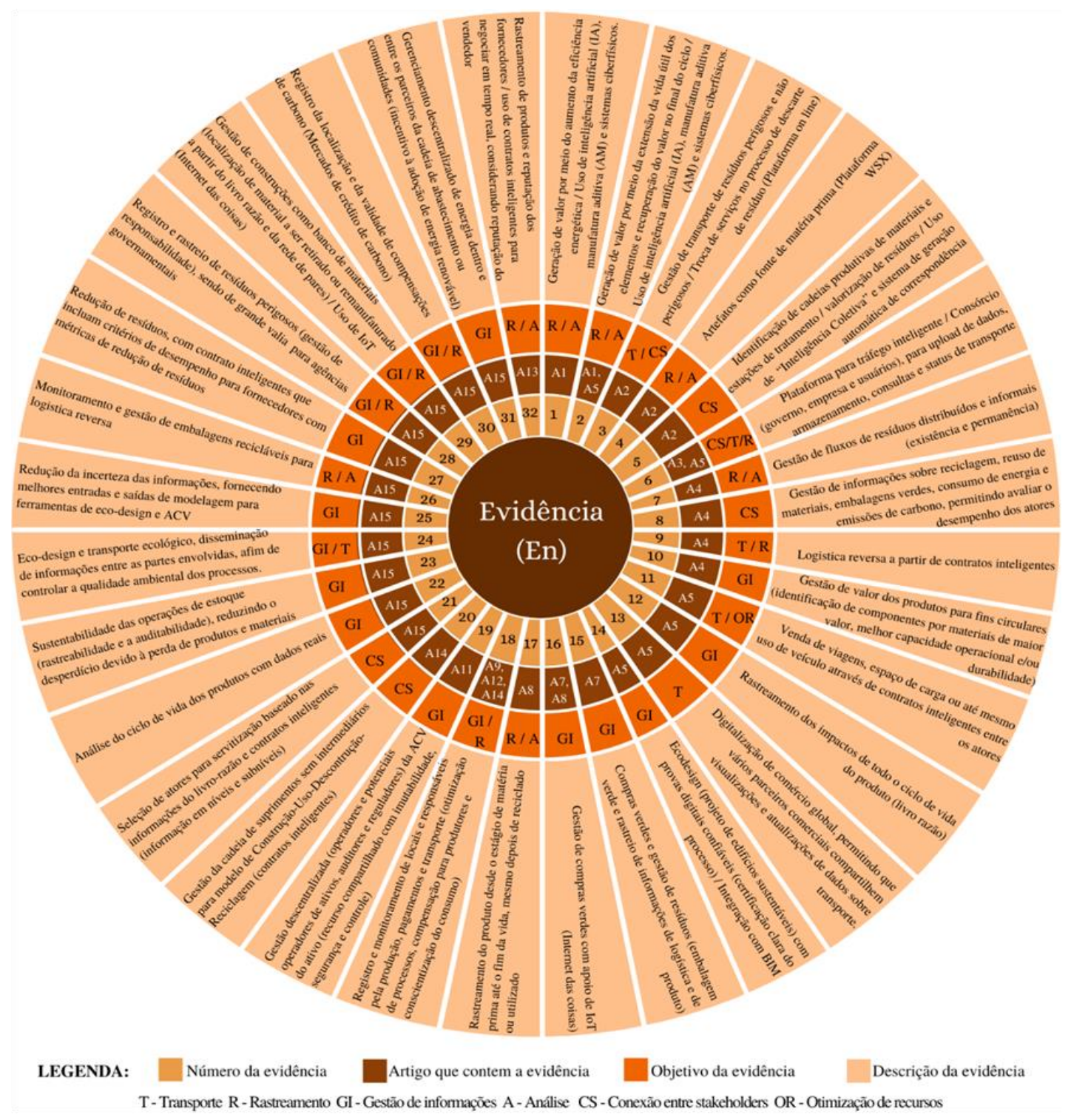

Fonte: autores

A análise das evidências também possibilitou sua correlação de acordo com os componentes- da blockchain e os processos da EC, a partir do uso de uma matriz de análise de conteúdo (Figura 4). A variação de cores apresentada na matriz busca ressaltar as células com maior quantidade de evidências identificadas (tom mais escuro), das células com menor quantidade (tom mais claro). A matriz permite identificar que a fase do planejamento e projeto é a que concentra o maior número de evidencias categorizadas (17 evidências que 
representam 53,1\% do total e estão alocadas 35 vezes na matriz). Esta fase inclui 4 processos (Eco desenho, AIA, ACV e servitização). O processo da EC com mais evidências, por sua vez, é o de transporte, com $28,1 \%$ do total (9 evidências), seguido de despejo, com 21,9\% (7 evidências). A concentração de evidências encontra-se nessas 3 fases (Planejamento, Produção e Gestão do RCD). Um ponto importante a ser mencionado é que o processo de manutenção não apresentou evidências e as fases de execução e de uso foram as fases com menos evidências, com 2 (6,2\% do total) e 1 (3,1\% do total), respectivamente. Já com relação aos processos da BT, os componentes de livro-razão e rede de pares são os componentes com mais evidências encontradas, com 46,9\% (15 evidências, alocadas 19 vezes na matriz) e 40,6\% (13 evidências, alocadas 17 vezes na matriz), respectivamente.

Figura 4 - Matriz de análise de conteúdo

\begin{tabular}{|c|c|c|c|c|c|c|c|c|c|c|c|c|}
\hline \multirow{3}{*}{$\begin{array}{l}\text { COMPONENTES DA } \\
\text { BLOCKCHAIN }\end{array}$} & \multicolumn{12}{|c|}{ PROCESSOS DA EC } \\
\hline & \multirow{2}{*}{\begin{tabular}{|c|}
$\begin{array}{c}\text { Fase de } \\
\text { produção }\end{array}$ \\
Transporte \\
\end{tabular}} & \multicolumn{4}{|c|}{ Fase de planejamento e projeto } & \multirow{2}{*}{\begin{tabular}{|c|}
$\begin{array}{c}\text { Fase de } \\
\text { execução }\end{array}$ \\
$\begin{array}{c}\text { Reuso no } \\
\text { local }\end{array}$ \\
\end{tabular}} & \multicolumn{3}{|c|}{ Fase de uso } & \multicolumn{3}{|c|}{ Fase de gestão do RCD } \\
\hline & & \begin{tabular}{|c|} 
Eco \\
desenho
\end{tabular} & AIA & $\mathrm{ACV}$ & \begin{tabular}{|c|} 
Serviti- \\
zação
\end{tabular} & & $\begin{array}{c}\text { Manut } \\
\text { enção }\end{array}$ & \begin{tabular}{|l} 
Reab. \\
parcial
\end{tabular} & $\begin{array}{l}\text { Reab. } \\
\text { total }\end{array}$ & $\begin{array}{c}\text { Descons- } \\
\text { trução }\end{array}$ & \begin{tabular}{|c|} 
Valor. \\
energética
\end{tabular} & Despejo \\
\hline Livro Razão & $\begin{array}{c}\text { E18, E21, } \\
\text { E30, E31 } \\
\end{array}$ & $\begin{array}{c}\text { E14, } \\
\text { E26, E27 }\end{array}$ & E30 & $\begin{array}{l}\text { E12, E19, } \\
\text { E24, E27 }\end{array}$ & E22 & E8 & & & & & E32 & $\begin{array}{l}\text { E5, E25, } \\
\text { E30, E31 }\end{array}$ \\
\hline Rede de pares & $\begin{array}{c}\text { E6, E13, E18, } \\
\text { E30, E31 }\end{array}$ & E26, E27 & E30 & $\begin{array}{c}\text { E10, E17, } \\
\text { E27 }\end{array}$ & & & & & & & E32 & $\begin{array}{c}\text { E5, E7, E25, } \\
\text { E30, E31 }\end{array}$ \\
\hline Serviços de associação & E6, E30 & E26, E27 & E30 & E27 & & & & & & & E32 & E30 \\
\hline Contrato inteligente & E6, E11 & & & E19 & E23 & E13 & & & & E21 & & E29 \\
\hline Eventos & $\begin{array}{c}\text { E18, E21, } \\
\text { E30 }\end{array}$ & E26, E28 & E30 & E19, E28 & & & & & & & E32 & E30 \\
\hline $\begin{array}{c}\text { Gerenciamento de } \\
\text { sistemas }\end{array}$ & E3, E18, E30 & E26 & E20, E30 & E19 & & & & & & & & E3, E30 \\
\hline Integração de sistemas & E5, E21 & E15, E16 & & E2, E19 & E3 & & & $\mathrm{E} 4$ & E4 & & E1 & E3 \\
\hline
\end{tabular}

Fonte: autores

\subsection{Levantamento de dados}

O mapeamento dos processos da empresa selecionada identificou todas as etapas da gestão de resíduos (armazenamento, transporte e destinação final), e o método adotado para cada processo por tipo de resíduo (Figura 5). Identificou-se a existência de um controle básico de separação de resíduos na empresa, realizado através de um software de gestão para a indústria da construção civil e de uma planilha para rastreamento de informações referentes ao resíduo e as etapas de transporte e destinação final, atestadas por meio de CTR (Controle de transporte de resíduos) ou MTR (Manifesto do controle de resíduos) e CDF (Certidão de destinação final). No canteiro, para garantir a separação e armazenamento dos resíduos são utilizadas baías de descarte, caixas de coleta e caçambas estacionárias. A empresa demonstra buscar alternativas para melhorar a gestão de resíduos na obra, porém a falta de infraestrutura do município para recebimento e destinação adequada de alguns tipos de resíduo impacta negativamente no processo, visto que parte considerável dos materiais são destinados à aterros sanitários. Problemas evidenciados na etapa de armazenamento, como o acondicionamento conjunto de resíduos de gesso com resíduos Classe A, também prejudicam a melhora na gestão desses processos, pois impedem tecnicamente uma destinação final desses resíduos alinhada com os principios da economia circular.

Figura 5 - Mapeamento dos processos - canteiro de obras selecionado

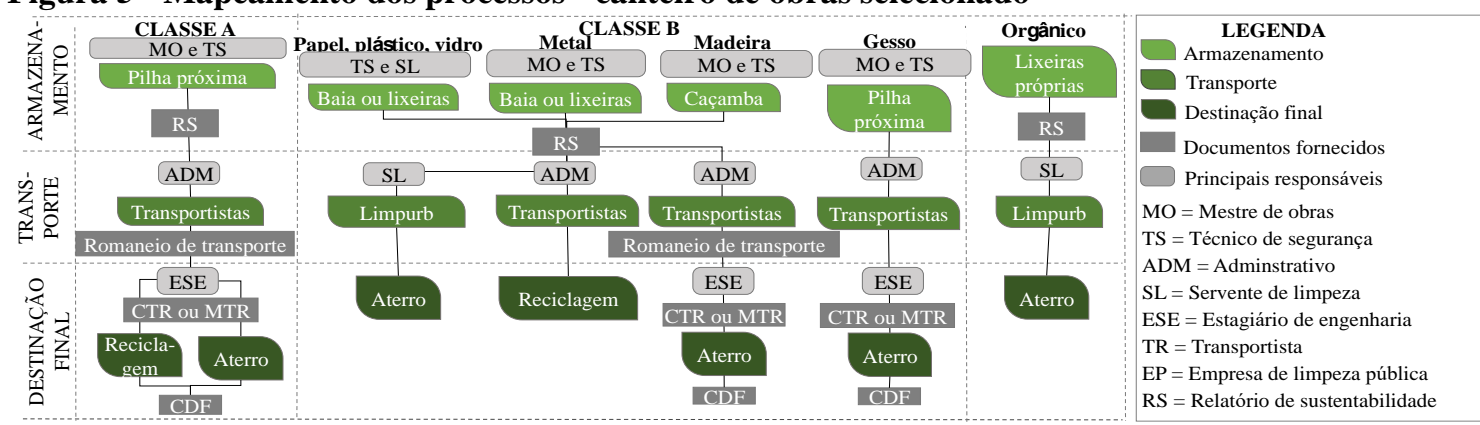

Fonte: autores 


\section{SISTEMA PROPOSTO}

Este estudo propõe o uso de BT para fomento da EC, através de uma proposta de uma plataforma on line para promoção e controle da gestão, compra e venda de RCD entre os agentes da cadeia produtiva do setor. Tendo em vista os resultados obtidos na bibliografia, o sistema busca resolver principalmente problemas relacionados à transporte $\mathrm{e}$ rastreamento de resíduos. Adota um conceito conceito SaaS (Software as a Service), onde a plataforma busca fortalecer a conexão entre os stakeholders relacionados. Determinamse, assim, 3 agentes atuantes na plataforma: o agente comprador / receptor (AC), o agente transportador (AT) e o agente vendedor / doador / gerador (AV). Como busca-se uma circularidade dos materiais, o AC e o AV podem ser virtualmente os mesmos agentes, não havendo diferenciação.

O AV coloca o seu material para venda ou doação e recebe propostas do AC. Este formato busca fomentar a identificação de cadeia de materiais e de gestão de resíduos, conforme observado na evidência E5, promovendo, se possível, simbiose industrial.

Uma vez que os termos do acordo estejam finalizados, há a contratação de uma empresa de transporte (AT), se aplicável, por meio do AV, e esta é autenticada pelo AC, para evitar fraudes. Essa contratação do AT poderá ser realizada de duas formas. A primeira é a disponibilização através de publicação em um canal geral para as empresas de transporte, e a segunda é a contratação de uma empresa anterior, de forma preferencial. Para melhor controle, uma versão do CTR / MTR ficará armazenado em uma tecnologia de nuvem com um hash (código criptografado sequencial) armazenado na blockchain via IPFS (InterPlanetary File Sistem, ou, em português, Sistema de Arquivos Interplanetário), que se trata de protocolo e rede projetada para criar um armazenamento associativo peer-to-peer endereçável ao conteúdo de armazenamento e compartilhamento, de hipermídia, num sistema de arquivos distribuído (NYALETEY, 2019). A CDF também é um documento a ser emitido e arquivado na plataforma. Tal processo permitirá o controle de alterações posteriores do documento.

Neste ponto, foram consideradas evidências identificadas na literatura como a necessidade de rastreamento (E32) e os sistemas evidenciados em E6 e E18, que indicam que os sistemas podem registrar e monitorar materiais a partir de transporte inteligente. Assim, a plataforma proposta possuirá sistema para upload de dados, armazenamento e consultas condicionais e do status de transporte dos resíduos em tempo real. O status e localização dos resíduos estará disponível aos agentes envolvidos, desde a etapa de coleta (emissão do CTR / MTR) até a etapa de reaproveitamento (emissão do CDF). Do mesmo modo, é prevista a gestão de informações sobre qualidade e eficiência dos processos, permitindo-se avaliar o desempenho dos atores (E8 e E32).

Todos os processos serão tratados a partir de contratos inteligentes, que poderão prever acordos que fomentem a servitização (E3 e E21) e a logística reversa (E9 e E26), tendo em vistas as características de rastreabilidade e imutabilidade proporcionadas pela BT. Para tal serão definidos parâmetros de compra, venda ou doação, que incluam tais aspectos.

A figura 6 mostra um fluxograma resumido dos processos da plataforma. Essa proposta é baseada nos processos realizados pelo canteiro objeto de análise.

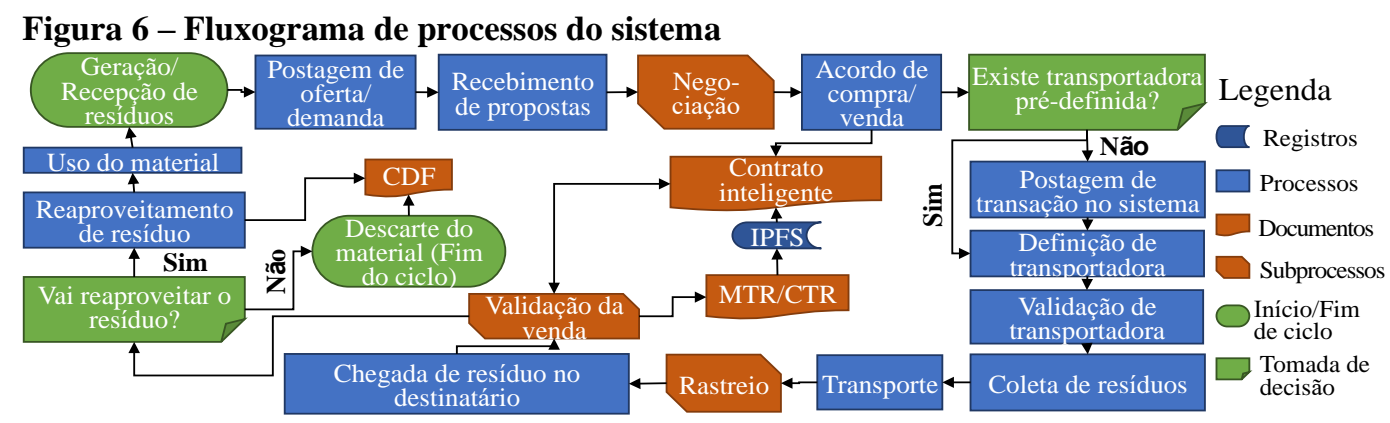

Fonte: autores 


\section{CONSIDERAÇÕES FINAIS}

A partir da revisão bibliográfica, observou-se grande quantidade de evidências relacionadas ao uso da BT para fomento da $\mathrm{EC}$ nos processos de transporte e despejo. Isso, aliado à falta de infraestrutura para gestão adequada de RCD verificada em campo, conduziu ao desenvolvimento de uma plataforma cujo objetivo principal fosse o controle da gestão, compra e venda de RCD. Através de um conceito SaaS (Software as a Service), a plataforma proporciona a conexão entre geradores, transportadores e receptores de resíduo, promovendo eventuais oportunidades de negócios circulares dentro da própria indústria, fomentando, consequentemente, a simbiose industrial. O uso de BT se destaca por oferecer contratos e registros rastreáveis, auditáveis e imutáveis que viabilizam ações de logística reversa e servitização entre os stakeholders, que permitem o controle do transporte em tempo real do resíduo, e que proporcionem a verificação da destinação apropriada dos resíduos e da qualidade e eficiência dos atores.

Como futuros passos desta pesquisa, o sistema proposto será construído e testado, avaliando, assim, o impacto dele no processo. Gestão de valor dos produtos (identificação de componentes por materiais de maior valor, melhor capacidade e/ou durabilidade), registro e rastreamento de todo o ciclo de vida dos materiais, gestão para futura desmaterialização (construção como banco de materiais) ou ACV podem ser objetivos a serem avaliados para implementação em etapa futura, com vistas a aumentar ainda mais o potencial da plataforma para fomentar a EC na indústria da construção.

\section{AGRADECIMENTOS}

Ao PIBIC (Programa Institucional de Bolsas de Iniciação Científica) / UFBA.

\section{REFERÊNCIAS}

ABRELPE. ASSOCIAÇÃO BRASILEIRA DAS EMPRESAS DE LIMPEZA PÚBLICA E RESÍDUOS ESPECIAIS. Panorama dos resíduos sólidos no Brasil 2018/2019. Relatório de 2019. São Paulo: ABRELPE, 2019.

AGOSTINHO, H. L.; GRANJA, Ariovaldo D.. Comparação de Modelos Contratuais na Construção Civil: Um Mapeamento Sistemático de Literatura. In: ENTAC, 16., 2016, São Paulo. Anais. Porto Alegre: ANTAC, 2016.

ALEXANDRIS, G. et al. Blockchains as Enablers for Auditing Cooperative Circular Economy Networks. IN: 23RD IEEE CAMAD, Proceedings... Barcelona: IEEE, 2018.

CASADO-VARA, R. et al. How blockchain improves the supply chain: case study alimentary supply chain. Procedia Computer Science, v. 134, 2018.

SCHMIDT, C. G.; WAGNER, S. M. Blockchain and supply chain relations: A transaction cost theory perspective. Jour. of Purch. and Sup. Manag.,v. 25, n. 4, 2019.

CIOFFI, R. et al. Smart Manufacturing Systems and Applied Industrial Technologies for a Sustainable Industry: A Systematic Literature Review. Appl. Sci., v. 10, n.8, 2020.

CONAMA. CONGRESSO NACIONAL DEL MEIO AMBIENTE. Economía Circular en el sector de la construcción. Madrid: CONAMA, 2018.

CONFORTO, R. WSX-European waste services exchange, instrument to start the transition towards circular economy. Proc. Envir. Sci., Eng. and Manag., v. 6, 2019.

DIAS, R. P. N. Análise de plataformas Blockchain. 2019. Dissertação de mestrado Universidade de Coimbra, Coimbra, 2019.

DU, X.; GAO, Y.; WU, C.; WANG, R.; BI, D. Blockchain-Based Intelligent Transportation: A Sustainable GCU Application System. Jour. of Adv. Transp., v. 2020, 2020. 
GHISELliNI, P.; CIALANI, C.; ULGIATI, S. A review on circular economy: the expected transition to a balanced interplay of environmental and economic systems. Journal of Cleaner Production, v. 114, 2016.

HULLEY, S. B. et al. Delineando a pesquisa clínica. $4^{\text {a }}$ edição. Artmed Editora, 2015.

KORHONEN, J.; HONKASALO, A.; SEPPÄLÄ, J. Circular Economy: the concept and its limitations. Ecological Economics, v. 143, 2018.

KOUHIZADEH, M.; ZHU, Q.; SARKIS, J. Blockchain and the circular economy: potential tensions and critical reflections from practice. Prod. Plan. \& Cont., v. 31, n.11-12, 2020.

KOUHIZADEH, M.; SARKIS, J. Blockchain Practices, Potentials, and Perspectives in Greening Supply Chains. Sustainability, v. 10, n. 10, 2018.

KOUHIZADEH, M.; SARKIS, J.; ZHU, Q. At the Nexus of Blockchain Technology, the Circular Economy, and Product Deletion. Applied Sciences, v. 9, n. 8, 2019.

NGUYEN, B. et. al. Hyper-ledger: Protocol specification. Hyperledger Org, 2018.

NYALETEY, E. et al. "BlockIPFS - Blockchain-Enabled Interplanetary File System for Forensic and Trusted Data Traceability," 2019 IEEE International Conference on Blockchain (Blockchain), 2019, pp. 18-25.

PAI, M. et al. Clinical Research Methods Systematic reviews and meta-analyses: An illustrated, step-by-step guide. National Medical Journal of India, v. 17, n. 2, 2004.

PERERA, S.; NANAYAKKARAA, S.; RODRIGO, M.; SENARATNEA, S.; WEINANDB, R. Blockchain technology: Is it hype or real in the construction industry? Journal of Industrial Information Integration. v. 17. 2020.

RAMAKRISHNA, S. et al. Emerging Industrial Revolution: Symbiosis of Industry 4.0 and Circular Economy: The Role of Universities. Sci., Tech. and Soc., v. 25, 2020.

RANE, S.B.; THAKKER, S.V. Green procurement process model based on blockchainIoT integrated architecture for a sustainable business. Management of Environmental Quality, v. 31 n. 3, 2019.

RIBEIRO, F. M.; KRUGLIANSKAS, I. A Economia Circular no contexto europeu: conceito e potenciais de contribuição na modernização das políticas de resíduos sólidos. IN: XVIENGEMA, 2014, São Paulo. Anais. São Paulo: ENGEMA, 2014.

RODRIGO, M. N. N.; et al. Potential Application of Blockchain Technology for Embodied Carbon Estimating in Construction Supply Chains. Build., v. 10, n. 8, 2020.

ROQUE, R. A. L.; PIERRI, A. C. Intelligent use of natural resources and sustainability in civil construction. Research, Society And Development. v. 8, n. 2, 2019.

SHOKOUHYAR, S.; PAHLEVANI, N.; SADEGHI, M. M. F. Scenario analysis of smart, sustainable supply chain on the basis of a fuzzy cognitive map. Management Research Review, v. 43 n. 4, 2019.

SWAN, M. Blockchain: Blueprint for a New Economy. O'Reilly Media Inc: USA, 2015.

TREIBLMAIER, H. The impact of the blockchain on the supply chain: a theory-based research framework and a call for action. Supply Chain Management, v. 23, n. 6, 2018.

$\mathrm{XU}, \mathrm{X}$. et al. The blockchain as a software connector. 13th Working IEEE/IFIPConf. on Software Architecture (WICSA), 2016, pg. 182-191.

WANG M.; WANG, C. C.; SEPASGOZAR S.; ZLATANOVA, S. A Systematic Review of Digital Technology Adoption in Off-Site Construction: Current Status and Future Direction towards Industry 4.0. Buildings, v. 10, n. 11, 2020. 